\title{
L'éloge paradoxal dans la littérature française contemporaine
}

Paradoxical Praise in Contemporary French Literature

\section{Christelle Reggiani}

\section{OpenEdition}

Journals

Édition électronique

URL : https://journals.openedition.org/recherchestravaux/4245

DOI : 10.4000/recherchestravaux.4245

ISSN : 1969-6434

Éditeur

UGA Éditions/Université Grenoble Alpes

Édition imprimée

ISBN : 978-2-37747-326-7

ISSN : 0151-1874

\section{Référence électronique}

Christelle Reggiani, «L'éloge paradoxal dans la littérature française contemporaine », Recherches \&

Travaux [En ligne], 99 | 2021, mis en ligne le 08 décembre 2021, consulté le 11 décembre 2021. URL http://journals.openedition.org/recherchestravaux/4245;DOI : https://doi.org/10.4000/ recherchestravaux.4245

Ce document a été généré automatiquement le 11 décembre 2021.

(C) Recherches \& Travaux 


\title{
L'éloge paradoxal dans la littérature française contemporaine
}

\author{
Paradoxical Praise in Contemporary French Literature
}

\section{Christelle Reggiani}

1 Dans la mesure où l'éloge paradoxal renvoie à une époque en principe révolue du discours public, on commencera par une brève mise au point historique afin de mieux appréhender ce genre singulier de l'«ancienne rhétorique ${ }^{1}$ ", dont les objets consistaient en des réalités précisément supposées indignes d'éloge, du fait de leur insignifiance (un insecte, la poussière, la fumée) ou bien du caractère négatif qui leur était communément attaché (Hélène, un tyran, la calvitie ${ }^{2}$ ).

2 Désigné par son opposition, ou du moins son décalage, par rapport à l'opinion usuelle (doxa), l'éloge paradoxal se situe également en marge du système élaboré par la Rhétorique d'Aristote, rapportant toute prise de parole publique à une détermination sociale qui en définit la fonction. Émergent alors trois genres de discours, que distingue avant tout l'ancrage institutionnel fondant leur pertinence sociale : le genre judiciaire, rassemblant les discours d'accusation et de défense prononcés dans l'enceinte des tribunaux ; le genre délibératif, qui englobe les discours politiques, proposant (de façon positive ou négative) tel ou tel type d'action ; le genre épidictique enfin, que définissent les discours, plus hétérogènes, énoncés sur les tribunes, éphémères ou permanentes, accueillant les commémorations publiques ${ }^{3}$. Ce cadre civique - le cas échéant élargi à l'ensemble de la nation - suscite généralement des discours réaffirmant à l'occasion de l'éloge de tel individu, de tel groupe, les valeurs qui fondent la communauté à laquelle appartiennent l'orateur et ses auditeurs. L'intérêt de la répétition tient alors à l'effet qui lui est prêté : la reformulation des valeurs - en son double sens de réitération et de variation - étant supposée renforcer la concorde sociale, comme sentiment et comme comportement (que le premier ait une action sur le second soutient en tout cas la prise de parole de l'orateur). Toutes choses égales par ailleurs, la réussite pratique de l'éloge consiste en somme en l'expérience, par le public, de sa propre cohésion : il y a là une finalité sociale qui, pour être plus difficile à saisir sans doute que dans les enceintes du tribunal ou de l'assemblée politique, n'est en pas moins agissante ${ }^{4}$. Cet aspect explique 
du reste la relative rareté des discours de blâme : si l'histoire n'est malheureusement pas avare d'exemples de mobilisations collectives, plus ou moins durables, contre des individus ou des groupes faisant figure de boucs émissaires, l'adhésion négative est par définition incapable de fonder une communauté sur des éléments positifs, quelle que soit leur nature.

3 C'est dire que l'éloquence épidictique est profondément endoxale : réunissant une communauté autour des valeurs qu'elle se reconnaît déjà, l'éloge, intrinsèquement conservateur, persuade moins qu'il ne renforce le consentement au monde tel qu'il est. Dans les termes de Perelman :

La conception même de ce genre oratoire [...] le fera pratiquer de préférence par ceux qui, dans une société, défendent les valeurs traditionnelles, les valeurs admises, celles qui sont l'objet de l'éducation [...]. Ne craignant pas la contradiction, l'orateur y transforme facilement en valeurs universelles, sinon en vérités éternelles, ce qui, grâce à l'unanimité sociale, a acquis de la consistance ${ }^{5}$.

Ainsi parfaitement approprié aux régimes autoritaires, le genre épidictique est notamment cultivé, sous l'Empire, par les orateurs de la Seconde Sophistique, au II ${ }^{e}$ siècle de notre ère : si ces professeurs d'éloquence élaborent dans leurs écoles des exercices progressifs variés, mobilisant les trois genres aristotéliciens au service de causes entièrement fictives ${ }^{6}$, la rhétorique épidictique - en principe dégagée de tout enjeu politique direct - est particulièrement sollicitée. Dans ce cadre, la tension qui fonde l'éloge paradoxal, censé heurter l'opinion commune, donne à la virtuosité des orateurs et de leurs élèves l'occasion de son plein déploiement, dans des morceaux de bravoure d'autant plus spectaculaires que leur objet est inattendu. Dans ces tours de force, l'épidictique retrouve le sens de son étymon, epideikvumi signifiant en grec « faire la démonstration, voire l'étalage, de son talent $»$ : ainsi de l'éloge du tabac qui ouvre le Dom Juan de Molière, ou de celui de Thomas Diafoirus à l'acte II du Malade imaginaire - pour emprunter quelques exemples à l'« âge de l'éloquence ${ }^{7}$ » que constitua à son tour le XVII ${ }^{\mathrm{e}}$ siècle français.

5 Autant dire que la forme semble datée, cantonnée à des temps révolus, antiques ou classiques. Or, cette éloquence apparemment désuète connaît une étonnante résurgence dans la littérature française contemporaine (en accordant à cet adjectif une valeur restreinte, qui étire le début du xxi ${ }^{e}$ siècle vers l'amont jusqu'à englober la fin du siècle dernier). Pour donner une première illustration du phénomène, on mentionnera très vite trois exemples (en guise de pointe d'iceberg), empruntés à deux textes choisis plutôt que d'autres parce que l'épidictique paradoxal constitue, quelle que soit son orientation, un leitmotiv dans l'économie générale de ces livres : la vitupération du chou-fleur et l'éloge de la fourmi dans L'Auteur et Moi d'Éric Chevillard, celui de la gibbosité dans Fragments de Lichtenberg de Pierre Senges ${ }^{8}$.

6 Il y a là une manière d'assumer l'anachronisme qui lui donne sa pleine évidence formelle, et manifeste ainsi la complexité, voire l'impureté temporelle du nouvel «âge de l'éloquence ${ }^{9}$ » contemporain - dont participe le présent volume. Si le mouvement rétrospectif qui alimente ce retour de flamme rhétorique suppose une discordance, l'écriture a cependant toute latitude pour jouer, ou non, de cet écart, qui ne donne pas nécessairement lieu à des désaccords stylistiques : l'impulsion mimétique au principe du pastiche, que sa finalité soit ironique ou sérieuse, occulte un décalage que l'insertion - dans une prose régie par d'autres modèles - de morceaux de bravoure oratoires (en l'occurrence épidictiques) accentue en revanche. De fait, la littérature contemporaine 
ne manque pas de discours investissant sérieusement un registre rhétorique dont ils assument pleinement l'orientation pragmatique - on pense par exemple aux manifestes féministes de Virginie Despentes et Chloé Delaume ${ }^{10}-$, mais ce sont des objets temporels complexes que l'on privilégiera dans les pages qui suivent, dans la mesure où leur " polychronie ${ }^{11}$ ", mettant en relief l'anachronisme de toute histoire de l'art, fait justement problème. On se propose en somme, en tentant de cerner plus précisément les contours du phénomène, d'interroger les enjeux d'une telle résurgence - qui n'a d'ailleurs rien d'une "survivance ${ }^{12}$ " puisque ce retour de l'éloge paradoxal dans la littérature française contemporaine ne suppose pas, de la part des écrivains, la lecture effective du corpus antique fondateur du genre.

\section{Histoire de la littérature récente (Olivier Cadiot)}

7 Si la veine épidictique de la littérature française actuelle semble trouver l'une de ses sources dans l'éloge funèbre de la mouche recueilli dans Écrire de Marguerite Duras (1993), le registre sérieux - qui est incontestablement celui du texte de Duras - y est cependant moins présent que le goût distancié pour une inspiration ironique où l'éloge paradoxal apparait en somme comme une dilatation - ou une amplification, pour le dire d'un terme rhétorique - de l'incongru ${ }^{13}$. Il faudrait relire ici l'œuvre d'Éric Chevillard dont certains livres enchaînent les morceaux de bravoure virtuoses au point d'offrir au lecteur un véritable feu d'artifice(s) rhétorique(s) prenant toutes les allures d'un hommage, jubilatoire, aux orateurs de la Seconde Sophistique. Lorsque le blâme véhément $\mathrm{du}$ chou-fleur - ou plus précisément du gratin de chou-fleur, que «l'auteur » déteste - qui forme le fil rouge de L'Auteur et Moi laisse place à une longue filature de fourmi ${ }^{14}$, le livre retrouve du reste une ressource topique des éloges sophistiques (l'être infime qu'est l'insecte figurant par excellence l'inconsistant support d'une performance oratoire paradoxale) :

La fourmi, voilà, existe à peine et pourtant ne redoute rien - devrait être écrasée par la seule idée de l'immensité du monde qui la surplombe, et pourtant non. Une vitalité à toute épreuve que nulle avanie ne menace. Six pattes, mais pas une seule pour se gratter dubitativement le front. Va de l'avant toujours et en tout lieu - qui lui barrera la route si l'éléphant ne le peut pas? [...] Si ton magnifique aplomb se lézarde entre tes orteils, elle passera par là. Tout est brèche qui n'est point béton pour la toute toute toute petite fourmi. S'insinue, s'immisce mieux que l'eau - suinterait-elle ? N'importe quoi ! Elle perle à la surface de toute chose ${ }^{15}$.

8 On mentionnera encore le motif obsédant de la poussière - en écho à l'éloge de Fronton? - dans Mentir d'Eugène Savitzkaya ${ }^{16}$, ou l'éloge de l'infirmité du personnage éponyme dans Fragments de Lichtenberg de Pierre Senges (Lichtenberg était bossu) :

La gibbosité debout est l'orgueil de l'infirmité ; la gibbosité assise est absence de leçon comme de vanité : la bosse ne s'exhibe plus, disparaît presque, sans se dissimuler pourtant. La gibbosité assise, c'est la paix retrouvée à partir du moment où, du fond de son fauteuil, l'homme tordu parvient à se réconcilier avec sa bosse, ne faire plus qu'un avec elle, comme cul et siège, et se conformer à sa propre silhouette comme s'il s'agissait de se résigner à sa propre caricature, et de lui trouver un certain cachet. Lichtenberg, ainsi, parvient à prendre place dans sa gibbosité comme dans le plus confortable des fauteuils, sans dire un mot, en se vouant au cosy, devenu le plus haut degré de la sagesse (provisoirement) ${ }^{17}$.

La liste pourrait être prolongée - d'autant plus facilement que ce goût de l'éloge a pris une consistance éditoriale dans une collection des éditions Gallimard $^{18}-$ et le corpus 
de toute façon sensiblement élargi si l'on observe que la longueur même de l'évocation, de la description, vaut éloge de la réalité à laquelle elle s'attarde ainsi - éloge paradoxal dès lors qu'un lecteur juge disproportionnée une telle attention. Fondé sur l'articulation de jugements de valeur - celui que suppose, ou feint de supposer, l'existence du discours contredisant en principe celui prêté à l'opinion commune-, l'éloge paradoxal ne peut qu'être un genre à géométrie variable. Pour s'en tenir ici à quelques exemples, pourraient ainsi lui être annexés certains au moins des Petits Traités de Pascal Quignard ${ }^{19}$ - d'ailleurs fin connaisseur de la littérature romaine et auteur, en particulier, d'une Rhétorique spéculative dont l'incipit cite Fronton ${ }^{20}-$, de même qu'un texte comme La Mélancolie de Zidane ${ }^{21}$ : Jean-Philippe Toussaint a beau être un supporter sérieux (en témoigne Football ${ }^{22}$ ), la plaquette qu'il consacre au coup de tête donné au défenseur italien Materazzi par le meneur de jeu de l'équipe de France lors de la finale de la Coupe du monde de 2006, et à l'expulsion qui s'ensuivit, peut bien être reçue, par quelques lecteurs au moins, comme un discours éminemment paradoxal au sein du champ littéraire.

10 Faisant ainsi la part belle au dérisoire, la littérature semble tenir un « discours sur le peu de réalité23 », participant d'une éloquence « infra-ordinaire ${ }^{24}$ » qui apparaît comme une forme exacerbée de l'écriture du quotidien qui détermine tout un pan de la littérature contemporaine.

11 Le phénomène a été précisément décrit par Michael Sheringham, qui en a établi la généalogie intellectuelle (dans les écrits de Henri Lefebvre, Roland Barthes, Michel de Certeau et Georges Perec ${ }^{25}$ ), et Kristin Ross en a proposé une archéologie convaincante dans l'espace français : la décolonisation aurait marqué la fin, pour ce pays, des grands récits politiques (et notamment du Grand Récit gaullien), en distinguant l'Histoire nationale de celle de l'Empire. Ce retrait objectif sur l'espace restreint du territoire national aurait très vite pris le sens d'un repli sur une intériorité éprouvée comme fin de l'Histoire: la "colonisation du quotidien ${ }^{26}$ » aurait alors relayé une aventure coloniale désormais refermée. Il y a là une dénégation dont l'adoption, exactement contemporaine, de «régimes d'historicité ${ }^{27}$ " synchroniques (ou tendant à la synchronicité) dans le domaine des sciences humaines - linguistique, histoire (avec la « longue durée » des Annales, qui est aussi une durée lente, puis la micro-histoire, venue d'Italie, du quotidien des gens de peu) - semble constituer la contrepartie scientifique.

S'accentue alors la démocratisation du roman telle que les travaux de Jacques Rancière l'ont mise au jour, rapportant l'attention égale que la prose moderne accorde aux divers éléments composant les univers fictionnels à l'affirmation du principe de représentation dans le champ politique ${ }^{28}$. C'est définir, dans la longue durée, une boucle de rétroaction complexe, où la représentation esthétique procéderait de déterminations politiques - largement externes, donc - qu'elle est susceptible de renforcer à son tour, la décolonisation constituant dans cette optique l'intervention d'une nouvelle causalité externe, de même orientation.

13 Ainsi largement cantonné à l'insignifiant, l'art contemporain de la prose se contenterait-il en somme, dans le sillage de Flaubert, de «livres sur rien »? Il est sûr que l'engagement des autrices et des auteurs actuels adopte le cas échéant des formes mineures - sans qu'il y ait là nul jugement de valeur -, témoignant sans doute d'une extinction délibérative redoublée par la marginalité sociale de l'écrivain, qui ne peut aujourd'hui prétendre qu'à parler depuis une tribune relativement confidentielle. 
Ce n'est pas à dire, pourtant, que l'«action restreinte ${ }^{29}$ » de la littérature soit absolument sans effet. On proposera alors de voir dans les modes divers de l'épidictique "infra-ordinaire» une manière pour les écrivains de négocier avec une telle restriction: qu'il s'agisse de l'éloquence en miettes de Nathalie Quintane, lançant ses fragments - brèves notations à propos de chaussures ou de Saint-Tropez ${ }^{30}$ - comme autant de pavés ${ }^{31}$, ou des éloges surprenants d'Éric Chevillard, balançant entre le loufoque et le dérisoire, l'écriture trouve des formes idéales, capables d'exprimer le désir de communication d'une littérature qui persiste à se vouloir « de bon conseil » (selon la définition que Benjamin propose du conte ${ }^{32}$ ) tout en lui conférant l'allure provocante du paradoxe. Dans cette façon de tenir à distance, ou plutôt de biais, le souci du politique, la littérature met en œuvre une éloquence potentielle, en assumant ironiquement l'ambition tout en lui donnant, sans trop de nostalgie, la forme adressée de l'incongruité. C'est proposer un "louvoiement ${ }^{33}$ » tactique, dont l'écart ne dessine bien entendu qu'une voie possible.

\section{Les deux erreurs de Descartes}

Les champs de bataille de l'histoire en offrent bien des exemples, de tels déplacements n'ont de valeur que s'ils s'inscrivent dans le cadre, plus global, d'une stratégie offensive ; s'agissant de littérature, on parlera, à la suite d'Éric Chevillard, de « contreattaque »:

Nous sommes obligés de nous entendre sur un sens commun, nous choisissons de vivre dans un monde d'impostures et de chimères. J'ignore si ce compromis est la solution la meilleure, mais je sais que le champ de la littérature s'ouvre au delà. Il est cet espace mental poétique, infini, où la vie se poursuit, développe ces formes larvaires interdites de séjour sur terre. La littérature, quand elle se refuse à n'être qu'une évocation nostalgique ou rancunière de l'enfance ou un compliment hyperbolique adressé à la femme, crée ce réel intangible où la conscience élargie vit des expériences inédites tout aussi (im)probables et intenses que celles que lui propose la réalité. Ce n'est pas un mince profit ${ }^{34}$.

Si l'œuvre de Chevillard se rapproche le cas échéant du Manifeste pour une réforme radicale du système en vigueur envisagé, dans Le Caoutchouc décidément, par le personnage de Furne ${ }^{35}$, la littérature contemporaine dans son ensemble n'est pas avare de textes qui se rattachent, plus ou moins directement, à ce mouvement d'insurrection littéraire.

On observera d'abord que nombre d'entre eux se signalent par l'importance remarquable qu'ils accordent aux animaux. Le phénomène caractérise déjà les éloges paradoxaux de l'Antiquité, on l'a vu, mais il acquiert ici une signification particulière, valant au fond double récusation du cartésianisme (ou, plus exactement, du cartésianisme imaginaire qu'engendre la réception commune de la pensée de Descartes).

18 Auteur d'un Discours de la méthode dénonçant avec virulence l'invention rhétorique des preuves, Descartes a également élaboré un dualisme philosophique ordinairement réduit à une série d'oppositions faisant système, entre l'esprit et le corps, la raison et l'émotion, l'homme et l'animal: c'est en somme contre cette "erreur", naguère exposée par un livre d'Antonio Damasio ${ }^{36}$, que s'élèverait la littérature lorsqu'elle plaide - sans renoncer à la rhétorique donc-pour un élargissement de la communauté des sujets ${ }^{37}$. 
On proposera de voir dans l'éloge funèbre de la mouche que Duras inclut dans Écrire, en 1993, une matrice de ce tournant doublement anti-cartésien de la littérature française contemporaine. Étonnant avant-courrier de l'attention à l'animal qui caractérise la culture actuelle, assez loin de la poésie de Ponge mais à la manière, étrange, dont Hiroshima mon amour semble rétrospectivement faire œuvre dès 1959 des thèmes mémoriels qui s'imposeront à la toute fin $\mathrm{du} \mathrm{xx}^{\mathrm{e}}$ siècle et plus encore au $\mathrm{XxI}^{\mathrm{e}}$ :

Ma présence faisait cette mort plus atroce encore. Je le savais et je suis restée. Pour voir. Voir comment cette mort progressivement envahirait la mouche. Et aussi essayer de voir d'où surgissait cette mort. Du dehors, ou de l'épaisseur du mur, ou $\mathrm{du}$ sol. De quelle nuit elle venait, de la terre ou du ciel, des forêts proches, ou d'un néant encore innommable, très proche peut-être, de moi peut-être qui essayais de retrouver les trajets de la mouche en train de passer dans l'éternité. [...]

La mort d'une mouche, c'est la mort. C'est la mort en marche vers une certaine fin du monde, qui étend le champ du sommeil dernier. [...] Ce n'est pas grave mais c'est un événement à lui seul, total, d'un sens énorme : d'un sens inaccessible et d'une étendue sans limites. J'ai pensé aux Juifs. J'ai haï l'Allemagne comme aux premiers jours de la guerre, de tout mon corps, de toute ma force. [...]

Cette précision de l'heure à laquelle elle était morte faisait que la mouche avait eu des funérailles secrètes. Vingt ans après sa mort, la preuve en est faite ici, on parle d'elle encore. [...]

Celle-là, celle que j'avais vue, moi, elle était morte. Lentement. Elle s'était débattue jusqu'au dernier soubresaut. Et puis elle avait cédé. Ça a peut-être duré entre cinq et huit minutes. Ça avait été long. C'était un moment d'absolue frayeur. Et ça avait été le départ de la mort vers d'autres cieux, d'autres planètes, d'autres lieux.

Je voulais me sauver et je me disais en même temps qu'il me fallait regarder vers ce bruit par terre, pour quand même avoir entendu, une fois, ce bruit de flambée de bois vert de la mort d'une mouche ordinaire. [...]

Autour de nous, tout écrit, c'est ça qu'il faut arriver à percevoir, tout écrit, la mouche, elle, elle écrit, sur les murs, elle a beaucoup écrit dans la lumière de la grande salle, réfractée par l'étang. Elle pourrait tenir dans une page entière, l'écriture de la mouche. Alors elle serait une écriture. Du moment qu'elle pourrait l'être, elle est déjà une écriture. Un jour, peut-être, au cours des siècles à venir, on lirait cette écriture, elle serait déchiffrée elle aussi, et traduite. Et l'immensité d'un poème illisible se déploierait dans le ciel. [...]

On peut aussi ne pas écrire, oublier une mouche. Seulement la regarder. Voir comme à son tour, elle se débattait, d'une façon terrible et comptabilisée dans un ciel inconnu et de rien.

Voilà, c'est tout ${ }^{38}$.

La mouche est cet être infime et méprisé - qui est aussi la mouche des vanités - dont Duras élargit la mort «infra-ordinaire » aux dimensions de l'univers, sans ironie ni allégorie. Et si l'agonie de la mouche ne représente pas, mais incarne, à sa très modeste échelle, non seulement le drame de la mort personnelle mais encore la tragédie collective des guerres et des massacres du $\mathrm{xx}^{\mathrm{e}}$ siècle, c'est qu'elle ressortit déjà en soi à l'écriture, participant ainsi d'une sémiologie avant même que la prose de Duras ne s'en saisisse. C'est pourquoi cette minuscule « réfraction » d'un fragment du grand livre du monde se souvient sans doute moins de l'équivalence, posée par le «Coup de dés » de Mallarmé, entre la page imprimée et le ciel étoilé, qu'elle ne fait écho aux craquelures des carapaces de tortues que les devins chinois brûlaient pour y lire des signes qui représentent aussi l'origine de l'écriture. Inscrivant ainsi à même le monde une écriture qui lui demeure immanente, les volètements de l'insecte mourant sollicitent une éloquence qui en soit le prolongement : une oraison funèbre certes dépouillée de l'apparat du Grand Siècle mais cependant capable d'ériger ces «funérailles » en effet 
offertes à la mouche en un tombeau de l'humaine condition. De cet «infra-ordinaire » le plus dérisoire naît le sublime: c'est retrouver, au sein du genre épidictique, le "sublime du lieu commun » que Francis Goyet assignait plutôt au judiciaire, expliquant que le dépassement du cas particulier en discussion vers les grands principes fondant la communauté comme telle engendrait en lui-même le sublime ${ }^{39}$.

21 Pour autant, le bestiaire des écrivains contemporains ne relève pas forcément du pathétique - au sens antique de la catégorie (c'est bien par l'émotion que l'oraison funèbre de Duras saisit son lecteur) -, même si cette tonalité y demeure le cas échéant présente, ainsi dans Anima de Wajdi Mouawad, livre d'une extrême violence qui juxtapose les discours et les pensées d'une série d'animaux ${ }^{40}$.

À un tout autre registre donc, on mentionnera par exemple - un exemple choisi parmi bien d'autres possibles - ce morceau de bravoure extrait d'Achab de Pierre Senges, « séquelle » du Moby Dick (1851) de Melville :

Être baleine : le sentiment d'être immense, un sentiment immense lui aussi, lent à venir, se propageant pendant des jours de la queue à la tête le long d'un corps dont rien, au fond, ne saurait faire le tour [...]. Il lui arrive de se croire fine et maigre, faite d'une seule tranche, comme une page, et de flotter ainsi plus librement: elle a pu se figurer creuse et compartimentée, elle s'est comparée à des épaves de caravelles, décousues, hérissées mais superbes (sa jalousie est récurrente) [...]. [...] sur terre, elle le sait pertinemment, elle s'écroulerait sous son poids, elle finirait par être lugubre, [...] elle adopterait par mimétisme la morosité des vaches : sans doute élégante, mais inébranlable dans sa tristesse ; sous l'eau, une baleine est pesante, on en connaît pourtant qui ont la grâce d'un impondérable ravioli, à point dans son bouillon ${ }^{41}$.

Si la baleine était déjà un objet littéraire - répertoriant les usages humains des différentes parties de son corps, le chef-d'œuvre de Melville y avait ajouté cette transmutation-là -, Pierre Senges a donné à cette attention portée à l'animal un tour plus universel dans Zoophile contant fleurette, qui réunit une collection de 99 textes brefs, entre déclaration d'amour et kamasutra zoophile. Pour mieux mesurer les effets de ce resserrement à l'aune de l'ampleur des éloges paradoxaux, on en citera quelques-uns, qui seront l'occasion de retrouver des animaux déjà croisés :

7. La puce : pas la plus belle des histoires, en tout cas la plus minutieuse - je ne dis pas ça pour me vanter. [...]

33. La mouche: encore maintenant, il est presque impossible de dire où, et comment, et combien de temps, et dans quelle posture, et si cette nervosité suivie d'abattement était bel et bien des noces.

37. La baleine : il est étrange de savoir sa maîtresse si loin de l'endroit où on lui fait l'amour - quand la reconnaissance, si reconnaissance il y a, parvient à l'amant, c'est avec un délai qui fait croire un instant à des prouesses surnaturelles, ou au contraire l'échec complet ${ }^{42}$.

La déclaration anti-dualiste a beau être ironisée, elle n'en est pas moins formulée.

Il importe alors que l'animal ne parle pas : son existence littéraire sera donc affaire de représentation, c'est-à-dire de simulation - de son corps, de son comportement, voire de sa pensée ou de son discours ${ }^{43}$. Et qu'il soit objet ou sujet du propos, c'est une même surenchère rhétorique qui se trouve à l'œuvre - un excès exemplairement illustré par la facture d'Anima ${ }^{44}$, mais aussi par la prosopopée de la fourmi que rapporte L'Auteur et Moi :

Depuis le fond du caveau, Doryla lance le signal de l'attaque. Nous sommes prêtes, nous n'avons d'autre corps que notre armure de guerrières. La colonie déferle sur nos hôtes désemparés, nous avons pour nous la surprise, la vitesse, le nombre, nous 
vaporisons généreusement notre acide, soyez bénis, agneaux de Dieu! Nous leur brûlons les yeux. Ils tombent à genoux; le tamanoir sur le flanc agite frénétiquement ses pattes. La deuxième vague d'assaut les recouvre. Nos mandibules les déshabillent, l'étroit vêtement de peau souple cède aux entournures, nous savons en élargir les mailles. Mâchez bien, sœurettes, c'est de la viande de premier choix ${ }^{45}$ !

Là où la narratologie actuelle analyse des narrations non naturelles ${ }^{46}$, au sens où ces récits sont portés par des narrateurs dont la prise de parole relève elle-même de la fiction, l'ancienne rhétorique reconnaissait en effet des prosopopées. Si l'une des fins que s'assigne la littérature contemporaine est d'élaborer des espaces énonciatifs où ses voix fictives donnent la parole aux sans-voix (gens de peu, exilés, animaux), la prosopopée y figure alors comme une ressource centrale - retrouvant du reste, assez loin de la perspective herméneutique de Paul De $\mathrm{Man}^{47}$, la définition qu'en donnait, à la fin du XIX ${ }^{e}$ siècle, Pierre Fontanier (même si l'animal y manque) :

La Prosopopée [...] consiste à mettre en quelque sorte en scène, les absents, les morts, les êtres surnaturels, ou même les êtres inanimés ; à les faire agir, parler, répondre, ainsi qu'on l'entend ; [...] et cela, ou par feinte, ou sérieusement, suivant qu'on est ou qu'on n'est pas le maître de son imagination ${ }^{48}$.

En ce sens, la dépense spectaculaire que représentent les discours invraisemblables, prosopopées ou éloges paradoxaux - en raison même de l'écart qu'ils installent par rapport aux attentes habituelles -, ne manifesterait rien d'autre que le propre de la littérature : affaire de rythme, de figures, pour composer une prose sublime exhibant fièrement son artifice, à l'instar de la sophistication des orateurs grecs de l'Empire romain.

L'idée d'un retour du genre épidictique dans la littérature française contemporaine pouvait sembler elle-même paradoxale : on a tenté, à travers ces quelques exemples, de donner un peu de consistance à cette hypothèse d'une rencontre rhétorique - sans qu'il s'agisse pour autant de reprise. Parce qu'il fait état de la virtuosité spectaculaire de certains textes, ce parcours semble alors mettre au jour une sorte d'histrionisme de la « littérature récente» (pour reprendre l'expression d'olivier Cadiot $^{49}$ ) : le jugement ne serait pas dénué de pertinence, à condition toutefois de remarquer que l'excès indique ici l'ironie. Il faut d'ailleurs le formuler à l'inverse, car c'est bien la distanciation ironique qui paraît autoriser l'éclat rhétorique : dans l'éloge paradoxal, l'éloquence revient en farce.

Le caractère ironique de ce nouvel âge de l'éloquence ne saurait conférer au discours de la littérature qu'une portée dérisoire, mais on défendra enfin l'idée, paradoxale à nouveau, que la vertu de cette humble politique tiendrait, pour une part au moins, à sa précarité même, exacerbée par la forme matérielle du livre - une modestie en effet de circonstance puisqu'elle se trouve exactement accordée à celle d'un être humain désormais dépossédé de son trône de « maître et possesseur de la nature » (Descartes). C'est autrement dit parce que la littérature elle-même constitue aujourd'hui une « zone à défendre ${ }^{50}$ » que la fragilité de sa voix, attachée par exemple à composer d'improbables bestiaires, est susceptible de sonner de la façon la plus juste. 


\section{NOTES}

1. R. Barthes, L'Ancienne Rhétorique. Aide-mémoire, Euvres complètes, Paris, Seuil, t. III, 2002.

2. On fait ici allusion à quelques exemples célèbres : Lucien a composé un éloge de la mouche ; Fronton de la poussière et de la fumée ; Gorgias a fait l'éloge d'Hélène ; Isocrate celui du tyran Busiris et Synésius celui de la calvitie. Voir P. Dandrey, L'Éloge paradoxal de Gorgias à Molière, Paris, P.U.F., 1997.

3. Voir Aristote, Rhétorique, livre I. Sur le genre épidictique, voir L. Pernot. La Rhétorique de l'éloge dans le monde gréco-romain, Paris, Institut d'études augustiniennes, 1993, coll. «Études augustiniennes ».

4. Voir Ch. Perelman et L. Olbrechts-Tyteca, Traité de l'argumentation. La Nouvelle Rhétorique [1958], Bruxelles, Éditions de l'université de Bruxelles, 2000, p. 62-68. Voir aussi L. Nicolas, « La fonction héroïque : parole épidictique et enjeux de qualification », Rhetorica, vol. 27, n 2, 2009, p. 115-141; ainsi que E. Danblon, «La rationalité du genre épidictique », dans M. Dominicy et M. Frédéric (dir.), La Mise en scène des valeurs: la rhétorique de l'éloge et du blâme, Lausanne, Delachaux et Niestlé, 2001, p. 19-47.

5. Traité de l'argumentation, ouvr. cité, p. 67-68.

6. Un monde parallèle que D. Russell a baptisé « sophistopolis " [Greek Declamation, Cambridge, Cambridge University Press, 1983]. Voir F. Desbordes, La Rhétorique antique: l'art de persuader, Paris, Hachette, 1996, p. 138-139, coll. « Langues et Civilisations anciennes ».

7. M. Fumaroli, L'Âge de l'éloquence. Rhétorique et "res literaria" de la Renaissance au seuil de l'époque classique [1980], Paris, Albin Michel, 1994, coll. « Bibliothèque de "L'Évolution de l'Humanité" ".

8. É. Chevillard, L'Auteur et moi, Paris, Minuit, 2012 ; P. Senges, Fragments de Lichtenberg, Paris, Verticales, 2008.

9. Dans la mesure où cet «empire rhétorique » (Perelman) s'exerce aussi bien sur les arts, la réflexion théorique et critique, que divers discours sociaux (parmi lesquels figure au premier chef celui de la pédagogie), il constitue un «moment" au sens où l'entend G. Philippe après Brunetière (voir en particulier Pourquoi le style change-t-il ?, Bruxelles, Les Impressions nouvelles, 2021).

10. V. Despentes, King Kong Théorie, Paris, Grasset, 2006 ; Ch. Delaume, Mes bien chères sœurs, Paris, Seuil, 2019.

11. G. Philippe, Pourquoi le style change-t-il ?, ouvr. cité, chap. VII. Voir aussi G. Didi-Huberman, Devant le temps. Histoire de l'art et anachronisme des images, Paris, Minuit, 2000, p. $22:$ « [...] l'histoire des images est une histoire d'objets temporellement impurs, complexes, surdéterminés. C'est donc une histoire d'objets polychroniques, d'objets hétérochroniques ou anachroniques. N'est-ce pas dire, déjà, que l'histoire de l'art est elle-même une discipline anachronique? " Cette complexité temporelle reconduit in fine à la "non-contemporanéité du contemporain » qui caractérise de façon générale l'histoire (voir R. Koselleck, Le Futur passé. Contribution à la sémantique des temps historiques [1979], Paris, Éditions de l'EHESS, 1990).

12. À moins de donner au terme l'acception qu'il prend dans les écrits esthétiques d'A. Warburg : voir G. Didi-Huberman, L'Image survivante. Histoire de l'art et temps des fantômes selon Aby Warburg, Paris, Minuit, 2002.

13. Voir P. Jourde, Empailler le toréador. L'Incongru dans la littérature française de Charles Nodier à Éric Chevillard, Paris, José Corti, 1999, coll. « Les essais ».

14. " Puis vint à passer sous mes yeux cette fourmi. [...] Son opiniâtreté aussitôt me fascina ; et j'attelais mon wagon au sien » (L'Auteur et Moi, ouvr. cité, p. 117-118). Le passage occupe une quarantaine de pages (p. 117-158).

15. Ibid., p. 198-199.

Recherches \& Travaux, 99 | 2021 
16. E. Savitzkaya, Mentir, Paris, Minuit, 1977.

17. Fragments de Lichtenberg, ouvr. cité, p. 111.

18. Créée en 2007, la collection de poche «Petit éloge » compte à ce jour 47 titres, parmi lesquels figurent par exemple Petit éloge de la bicyclette d'É. Fottorino (2007), Petit éloge de la peau de R. Detambel (2007), ou encore Petit éloge de la jalousie de G. Obiégly (2007).

19. P. Quignard, Petits traités [1990], Paris, Gallimard, 1997, coll. « Folio », deux volumes.

20. "J'appelle rhétorique spéculative la tradition lettrée antiphilosophique qui court sur toute l'histoire occidentale dès l'invention de la philosophie. J'en date l'avènement théorique, à Rome, en 139. Le théoricien en fut Fronton ». P. Quignard, Rhétorique spéculative, Paris, Calmann-Lévy, 1995, p. 11.

21. J.-Ph. Toussaint, La Mélancolie de Zidane, Paris, Minuit, 2006.

22. J.-Ph. Toussaint, Football, Paris, Minuit, 2015.

23. A. Breton, Introduction au discours sur le peu de réalité, Paris, Gallimard, 1927.

24. Le mot, forgé par P. Virilio, a été repris par G. Perec : voir notamment L'Infra-ordinaire, Paris, Seuil, 1989.

25. M. Sheringham, Everyday Life. Theories and Practices from Surrealism to the Present, Oxford, Oxford University Press, 2006. Traduit en français par M. Heck et J.-M. Hostiou sous le titre Traversées du quotidien. Des surrealistes aux postmodernes, Paris, P.U.F., 2013, coll. « Lignes d'art ».

26. K. Ross, Fast Cars, Clean Bodies. Decolonization and the Reordering of French Culture, Cambridge (Mass.), MIT Press, 1995. L'idée avait été avancée dès 1975 par le philosophe français P. Virilio: « Il faut nous retourner à la limite du monde pour contempler la banalité, car nous n'avons plus d'originalités à piller, nous n'avons plus de cultures étrangères à exploiter [...]» («La défaite des faits ", Cause commune, $\mathrm{n}^{\circ} 1$ [Le Pourrissement des sociétés], Paris, U.G.É., 1975, p. 260, coll. «10/18 »).

27. F. Hartog, Régimes d'historicité. Présentisme et expériences du temps, Paris, Seuil, 2003, coll. « La librairie du XXI ${ }^{\mathrm{e}}$ siècle ».

28. Voir J. Rancière, Politique de la littérature, Paris, Galilée, 2007, coll. " La philosophie en effet ».

29. S. Mallarmé, «L'action restreinte» [1895], Divagations [1897], dans Euvres complètes, t. II, Paris, Gallimard, 2003, p. 214-218, coll. « Bibliothèque de la Pléiade ».

30. N. Quintane, Chaussure, Paris, P.O.L, 1997 ; Saint-Tropez. Une Américaine, Paris, P.O.L, 2001.

31. Lorsque l'autrice publie en mai 2018 (cinquante ans après un autre mois de mai) un gros livre dans le sillage de «Nuit debout », la quatrième de couverture exploite la vertu politique de la syllepse : «Des gens s'assemblent, discutent, écrivent sur des murs, certains tapent dans des vitrines. En échange, on leur tape dessus, on les convoque au tribunal et, à l'occasion, on leur ôte un œil. C'est la vie démocratique. Alors, je me suis dit: Tiens, et si, pour une fois, je sortais un pavé ?» (N. Quintane, Un œil en moins, Paris, P.O.L, 2018).

32. W. Benjamin, « Le Conteur. Réflexions sur l'œuvre de Nicolas Leskov » [1936], CEuvres III, Paris, Gallimard, 2000, p. 119, coll. « Folio essais».

33. J'emprunte le terme à $\mathrm{Ch}$. McDonald, qui l'emploie, dans un contexte très différent, à propos des autrices du XVIII ${ }^{\mathrm{e}}$ siècle (« Le XVIII ${ }^{\mathrm{e}}$ siècle [1715-1793]», dans Martine Reid [dir.], Femmes et Littérature. Une histoire culturelle, t. I, Paris, Gallimard, 2020, p. 894, coll. « Folio essais »).

34. É. Chevillard, «Écrire pour contre-attaquer », Europe, $n^{\text {os }} 868-869,2001$, p. 326.

35. Son nom est celui de l'éditeur de La Comédie humaine : manière de rapporter cette virulence à l'entreprise totalisante de Balzac? (É. Chevillard, Le Caoutchouc décidément, Paris, Minuit, 1992, p. 18-19.)

36. A. Damasio, L'Erreur de Descartes : la raison des émotions [1994], Paris, Odile Jacob, 1995.

37. Sur la préoccupation écologique qui sous-tend cette inflexion littéraire, voir Pierre Schoentjes, Littérature et Écologie : le mur des abeilles, Paris, José Corti, 2020.

38. Marguerite Duras, Écrire [1993], Euvres complètes, t.IV, Paris, Gallimard, 2014, p. 859-862, coll. «Bibliothèque de la Pléiade». 
39. Voir F. Goyet, Le Sublime du "lieu commun». L'Invention rhétorique dans l'Antiquité et à la Renaissance, Paris, Honoré Champion, 1996, coll. «Bibliothèque de la Renaissance ».

40. W. Mouawad, Anima, Montréal/Arles, Leméac/Actes Sud, 2012.

41. P. Senges, Achab (séquelles), Paris, Verticales, 2015, p. 109.

42. P. Senges, Zoophile contant fleurette, Portiragnes, Cadex, 2012, p. 23, 32-34. Après un prélude emprunté à Giordano Bruno, la collection est précédée de ce titre : «Carnet tenu par le vénérable Noé pendant les jours du Déluge - puis au cours des mois suivants, de mémoire » (p. 21). La quatrième de couverture présente ainsi le projet du livre : «Et si Noé, pour pallier l'étroitesse de son Arche, s'était contenté d'offrir l'hospitalité aux seules femelles de toutes les espèces - se chargeant lui-même, par la suite, de la reproduction? Hypothèse avérée, puisque de cette expérience hors du commun [...] Noé a rapporté ces quelques notes : à la fois souvenirs de voyage, élégie amoureuse et manuel pratique. »

43. Voir S. Milcent-Lawson, « Zoographies : traitements linguistique et stylistique du point de vue animal en régime fictionnel », Revue des sciences humaines, $\mathrm{n}^{\circ} 328,2017$, p. 91-106.

44. Voir C. Badiou-Monferran et L. Denooz (dir.), Langues d'Anima. Écriture et histoire contemporaine dans l'œuvre de Wajdi Mouawad, Paris, Classiques Garnier, 2016, coll. « Rencontres ».

45. L'Auteur et Moi, ouvr. cité, p. 220-221. Le discours de la fourmi occupe trois pages du livre (p. 219-222).

46. Voir notamment B. Richardson, «De la narratologie non naturelle », dans S. Patron (dir.), Introduction à la narratologie postclassique: les nouvelles directions de la recherche sur le récit, Villeneuve-d'Ascq, Presses universitaires du Septentrion, 2018, p. 167-181.

47. P. De Man, The Rhetoric of Romanticism, New York, Columbia University Press, 1984.

48. P. Fontanier, Les Figures du discours [1821-1827], éd. Gérard Genette (1968), Paris, Flammarion, 1977, p. 404, coll. « Champs».

49. O. Cadiot, Histoire de la littérature récente. Tome I, Paris, P.O.L, 2016.

50. H. Merlin-Kajman, Lire dans la gueule du loup. Essai sur une zone à défendre, la littérature, Paris, Gallimard, 2016, coll. « NRF Essais ».

\section{RÉSUMÉS}

Appartenant à une époque en principe révolue du discours public, la forme de l'éloge paradoxal semble datée, cantonnée à l'Antiquité ou à l'âge classique. Or, cette éloquence apparemment désuète connaît une étonnante résurgence dans la littérature française contemporaine (en donnant à cet adjectif sa valeur usuelle dans les études littéraires, pour désigner la période qui débute avec les années 1980). En tentant de cerner plus précisément les contours du phénomène, cet article se propose d'interroger les enjeux d'une telle résurgence - qui ne suppose pas, d'ailleurs, la lecture effective par les écrivains du corpus antique fondateur du genre.

Belonging to a bygone era of public discourse, paradoxical praise seems confined to Antiquity or Early Modernity. However, this apparently obsolete eloquence is experiencing a surprising resurgence in contemporary French literature (giving contemporary its usual value in literary studies, to designate the period beginning with the 1980s). By attempting to define more precisely the contours of the phenomenon, this article proposes to question the stakes of such a resurgence (which does not presuppose the reading by contemporary writers of the ancient texts that founded the genre). 
INDEX

Mots-clés : éloquence épidictique, éloge paradoxal, littérature française contemporaine, animal, prosopopée

Keywords : epideictic eloquence, paradoxical praise, contemporary French literature, animal, prosopopeia

\section{AUTEUR}

\section{CHRISTELLE REGGIANI}

Sorbonne Université - STIH (EA 4509)

Christelle Reggiani est professeure de stylistique française à la faculté des lettres de Sorbonne Université.

Elle a notamment publié : Rhétoriques de la contrainte. Georges Perec, l'Oulipo, Saint-Pierre-du-Mont, Éditions InterUniversitaires, 1999 (rééd. Eurédit, 2013) ; Éloquence du roman. Rhétorique, littérature et politique aux XIX et XX $X^{e}$ siècles, Genève, Droz, 2008 ; L'Éternel et l'Éphémère. Temporalités dans l'œuvre de Georges Perec, Amsterdam/New York, Rodopi, 2010 ; Poétiques oulipiennes. La contrainte, le style, l'histoire, Genève, Droz, 2014. Elle a également dirigé l'édition des CEuvres de Georges Perec dans la «Bibliothèque de la Pléiade » des éditions Gallimard (2017). 